\title{
Diabetes and quality of life: A theoretical perspective
}

\author{
Komal Verma, Meenal Dadarwal ${ }^{1}$ \\ Assistant Professor, Amity Institute of Behavioural and Allied Sciences, Amity University, Jaipur, ${ }^{1}$ Assistant Manager (HR) Roopshree Exports \\ Ltd, Jaipur, Rajasthan, India
}

\section{A B S T R A C T}

With changing time and lifestyle, general health of population has been drastically affected, and deteriorating quality of life. The transition from a traditional to modern lifestyle and consumption of diets rich in fat and calories combined with a high level of mental stress has compounded the problem further. Diabetes is one of the consequences of such a lifestyle change. Over the past 30 years, the status of diabetes has changed from being considered a mild disorder of the elderly to one of the major causes of morbidity and mortality affecting the youth and middle-aged people. This paper aims to present review of association between diabetes and quality of life as quality of life is considered to be one of the major factors affecting diabetes.

Key words: Diabetes, management, quality of life

\section{INTRODUCTION}

A major noncommunicable disease in India with a widespread reach of affecting 62 million people is diabetes. The International Diabetes Federation (IDF) has raised a serious alarm for India by saying that nearly $52 \%$ of Indians are not aware that they are suffering from high blood sugar. In fact, this disease exposes the 44 lakh Indians aged between 20 and 79 years to stroke, kidney disease, amputations, heart damage, blindness, and nerve damage. ${ }^{[1]}$ Over the past 30 years, the status of diabetes has changed from being considered a mild disorder of the elderly to one of the major causes of morbidity and mortality affecting the youth and middleaged people.

The advancement of the economic and social front in India due to the spread of globalization, industrialization, and rapid urbanization has produced dramatic lifestyle

\begin{tabular}{|l|l|}
\hline \multicolumn{2}{|c|}{ Access this article online } \\
\hline Quick Response Code: & Website: \\
\hline & www.joshd.net \\
\hline & \\
\hline
\end{tabular}

changes leading to lifestyle-related diseases. The transition from a traditional to modern lifestyle and consumption of diets rich in fat and calories combined with a high level of mental stress has compounded the problem further. The "fast food culture" and "sedentarinism" are the main drivers of diabetes epidemic in India. Over the last few years, there has been shifted in the working population of India primarily from manual labor associated with the agriculture sector to physically less demanding office jobs. With the advent of highly addictive computer and video games, sedentarinism is now affecting the children and youth as they tend to spend more time in front of television sets or computers than playing outdoors. It was observed in India that the prevalence of diabetes was almost three times higher in individuals with light physical activity compared to those having heavy physical activity. ${ }^{[2]}$

\footnotetext{
This is an open access article distributed under the terms of the Creative Commons Attribution-NonCommercial-ShareAlike 3.0 License, which allows others to remix, tweak, and build upon the work non-commercially, as long as the author is credited and the new creations are licensed under the identical terms.

For reprints contact: reprints@medknow.com

How to cite this article: Verma K, Dadarwal M. Diabetes and quality of life: A theoretical perspective. J Soc Health Diabetes 2017;5:5-8.
} 
However, the other reasons identified for the spread of diabetes are as follows:

(a) Insulin resistance, i.e., metabolic syndrome wherein a cluster of factors consisting of abnormal fats, high blood pressure, obesity, and abnormal glucose levels is highly prevalent in Asian Indians.

(b) Stress which has manifested in our physical and mental health has a strong effect on increasing the Type 2 diabetes in the population.

(c) Family history is the major reason for rise of diabetes as the risk of child developing diabetes increases 50\% with parental history. ${ }^{[3]}$

This global epidemic has been classified into three major groups, namely, Type 1, Type 2, and gestational diabetes. The most common diabetic symptoms are intense hunger, weight issues, increased fatigue, blurred vision, irritability, skin infections, etc.

According to IDF, India will be home to 100 million diabetic people in the world. Thus, the upward project trend in the number of diabetic patients in the country emphasizes the importance of treatment and care for this disease. Therefore, patients' education and medical education are strongly recommended as the treatment for the disease and its associated risk factors which are highly complex. ${ }^{[4]}$ To maintain a correct metabolic control, the patient has to regulate blood sugar and undertake unpleasant medication which is usually accompanied along with this disease. The entire process makes the patient vulnerable to stress, thereby affecting quality of life. The World Health Organization defines quality of life as "an individual's perception of their position in life in the context of the culture and value systems in which they live, and in relation to their goals, expectations, standards, and concerns." ${ }^{[5]}$ According to Oort, it is a "broad ranging concept affected in a complex way to the person's physical health, psychological state, personal beliefs, social relationships, and their relationship to salient features of their environment." ${ }^{\text {[6] }}$

Thus, as identified by various studies, diabetes is a typical of chronic illnesses as the treatment is burdensome and the complications are life-threatening influencing the quality of life of the patient. The quality of life is not consistently associated with duration and type of diabetes. Complications of diabetes are the most important diseasespecific determinant of quality of life. People suffering from diabetes have to face the challenge of living with this chronic disease and manage it on day to day basis. Patients must fulfill the management demands every day; often, they have to make endless decisions for effectual efforts to reach the nondiabetic metabolic state. For example, taking insulin can substantially affect quality of life positively by reducing symptoms of high blood sugar, for instance, or negatively by increasing symptoms of low-blood sugar. The psychosocial toll of living with diabetes is often a heavy one, and this toll can often, in turn, affect self-care behavior and ultimately, long-term glycemic control, the risk of developing long-term complications, and quality of life.

Diabetic person has higher (24\%) prevalence of depression compared to the nondiabetic person (17\%) with significant differences in quality of life. ${ }^{[7]}$ Psychological distresses (depression, anxiety, and sleep disturbances) can have a negative impact on quality of life. The risk of depression is higher in diabetes, undiagnosed diabetes, and impaired glucose metabolism that has serious threat to quality of life. ${ }^{[8]}$ Thus, it should be given higher priority in prevention and control of diabetes.

In a study, researchers have identified predicators of quality of life of diabetic patients as lifestyle and personal and medical factors. Particularly, the study highlighted that the gender, age, marital status, trait, and income have higher score with life satisfaction. Further, it was noted that individuals who are nonsmoker and high on physical activity were found to be significantly related with better health in adults with diabetes. ${ }^{[9]}$ Another researcher forwarded similar conclusions regarding the impact of sociodemographic factors on quality of life among diabetic patients. ${ }^{[10]}$ Diabetes is related with lower quality of life scores in their sample. In their study, the mean scores of diabetic people were lower than the mean of nondiabetic people in all questions of quality of life. ${ }^{[11]}$ Furthermore, they emphasized that strategies should be designed to diagnose diabetes early which would not only prevent diabetic-related complications but also prevent corrosion of quality of life among the diabetic patients.

In a research, persons with or without diabetes were compared and their results were in consistence with the previous studies reporting worse quality of life for people with diabetes, especially regarding physical functioning and wellbeing. ${ }^{[12]}$ However, when the quality of life of diabetic people was compared to other chronic conditions (cardiac problems, epilepsy with seizures, arthritis, multiple sclerosis, stroke, and lung problems), it was found that diabetic people report better quality of life. They concluded that quality of life in people with diabetes could be improved by certain interventions, including the introduction of blood-glucose-lowering agents, changes in insulin delivery systems, and educational and counseling programs designed to facilitate the development of diabetes-specific coping skills. 
A study done in Australia on the individuals diagnosed with diabetes were found to be suffering from depression which has been correlated to their daily lives and psychological impact of the diagnosis of the diabetes. ${ }^{[13]}$ The disease imposes pragmatic constraints on the individuals besides making them feel disconnected from the mainstream. ${ }^{[14]}$ Further, the quality of life comprised fatigue, exhaustion, the need to go to bed early, fear of losing their job, and loss of interest may contribute to lost opportunities for social interaction. Many diabetics experience memory lapses which, frequently exacerbated by irritation and stress, may affect their ability to converse.

\section{QUALITY OF LIFE AND GENDER}

In the recent past, a number of studies have reported that diabetic men have better quality of life than among the women. Study on the Lebanese community found out that quality of life such as family, housework, and anxiety/depression are significantly affected by the gender differences. The female participants have been noted to be experiencing depression/anxiety more than their male counterparts. Females had lower quality of life which could be attributed to low physical activity and poorer social conditions. ${ }^{[15-20]}$ Research emphasized that men were more satisfied with their diabetic treatment regimen and missed fewer leisure activities due to diabetes as compared to the women. ${ }^{[21]}$

In a similar study done in Southern India, it had highlighted that males had higher quality of life scores than females. The quality of life score had significant association with socioeconomic status, education, and habitual physical activity. The study concluded that diabetes had an adverse effect on the quality of life of the patients. Regular meditation, creating self-help groups, physical activity, good glycemic control, improving female literacy, and avoidance of substance abuse may help to improve the quality of life. ${ }^{[22]}$

A related study on assessing variables affecting diabetes management among patients, family members, and health professionals was done, and it was found that quality of life is one of the major factors acting as a barrier as well as a facilitator in successful management of diabetes. ${ }^{[23]}$

\section{CONCLUSION}

The evidence above demonstrates a wealth of knowledge about diabetes and quality of life at global level. No doubt, there are various factors responsible for effective management of disease, but one of the important factors which came out in findings is quality of life. If quality of life of the individual suffering with diabetes is monitored regularly and consistent care is taken than this disease can be managed successfully leading to a better health and life resulting in prevention of this disease. Hence, after reviewing literature, it can be said that in future, more variable specific research is required, especially focusing on factors affecting quality of life which further affects physical or mental health of the individual. Thus, it would be appropriate to suggest that prevention of diabetes is need of an hour, and this objective can be achieved by empowering patients, health professionals, and family members regarding importance of good and healthy life.

Financial support and sponsorship

Nil.

\section{Conflicts of interest}

There are no conflicts of interest.

\section{REFERENCES}

1. International Diabetic Federation. Types of Diabetes; 2013. Available from: http://www.idf.org/types-diabetes [Last retrieved on 2015 Jun 10].

2. Mohan V, Sandeep S, Deepa R, Shah B, Varghese C. Epidemiology of type 2 diabetes: Indian scenario. Indian J Med Res 2007;125:217-30.

3. Gupta R. Diabetes in India: Current Status. Financial Express; 2008. Available from: http://www.archivehealthcare.financialexpress. com. [Last retrieved on 2015 Jun 16].

4. Redekop WK, Koopmanschap MA, Stolk RP, Rutten GE, Wolffenbuttel BH, Niessen LW. Health-related quality of life and treatment satisfaction in Dutch patients with type 2 diabetes. Diabetes Care 2002;25:458-63.

5. World Health Organization. Basic Documents. $43^{\text {rd }}$ ed. Geneva: World Health Organization; 2001.

6. Oort FJ. Using structural equation modeling to detect response shifts and true change. Qual Life Res 2005;14:587-98.

7. Goldney RD, Phillips PJ, Fisher LJ, Wilson DH. Diabetes, depression, and quality of life: A population study. Diabetes Care 2004;27:1066-70.

8. Nouwen A, Nefs G, Caramlau I, Connock M, Winkley K, Lloyd CE, et al. Prevalence of depression in individuals with impaired glucose metabolism or undiagnosed diabetes: A systematic review and meta-analysis of the European Depression in Diabetes (EDID) Research Consortium. Diabetes Care 2011;34:752-62.

9. Imayama I, Plotnikoff RC, Courneya KS, Johnson JA. Determinants of quality of life in adults with type 1 and type 2 diabetes. Health Qual Life Outcomes 2011;9:115.

10. Issa B, Baiyewu O. Quality of life of patients with diabetes mellitus in a Nigerian teaching hospital. Hong Kong J Psychiatry 2007;16:27-33.

11. Thommasen $\mathrm{H}$, Zhang $\mathrm{W}$. Health-related quality of life and type 2 diabetes: A study of people living in the Bella Coola Valley. BC Med J 2006;48:272-8.

12. Rubin RR, Peyrot M. Quality of life and diabetes. Diabetes Metab Res Rev 1999;15:205-18. 
13. Manderson L, Kokanovic R. "Worried all the time": Distress and the circumstances of everyday life among immigrant Australians with type 2 diabetes. Chronic IIIn 2009;5:21-32.

14. Aikens JE, Perkins DW, Piette JD, Lipton B. Association between depression and concurrent type 2 diabetes outcomes varies by diabetes regimen. Diabet Med 2008;25:1324-9.

15. Mustapha W, Hossain Z, Loughlin K. Management and impact of diabetes on quality of life among the Lebanese community of Sydney: A quantitative study. J Diabetes Metab 2014;5:329-428.

16. Vallis T, Higgins I, Edwards L, Murray A, Scott L. The role of diabetes education in maintaining lifestyle changes. Can $\mathrm{J}$ Diabetes 2005;29:193-202.

17. Miksch A, Hermann K, Trieschmann J, Roelz A, Heiderhoff M, Laux G, et al. Gender-specific differences in quality of life of patients with type 2 diabetes with or without participating in DMP. Gesundheitswesen 2008;70:250-5.
18. Tankova T, Dakovska G, Koev D. Education and quality of life in diabetic patients. Patient Educ Couns 2004;53:285-90.

19. Verbrugge LM. Sex differentials in health. Public Health Rep 1982;97:417-37.

20. Sharpe PA, Clark NM, Janz NK. Differences in the impact and management of heart disease between older women and men. Women Health 1991;17:25-43.

21. Rubin RR, Peyrot M. Men and diabetes: Psychosocial and behavioral issues. Diabetes Spectr 1998;11:81-7.

22. Mathew A, Anusree T, Mathew A, Archana S. Quality of life among type 2 diabetes mellitus patients in South India: A descriptive study. Am Int J Res Humanit Arts Soc Sci 2014;215:197-200.

23. Balhara YP, Kalra S, John M, Baruah MP, Sahay R, Bantwal G, et al. Quality of life of people with diabetes: Second diabetes attitudes wishes and needs (DAWN2) study — Indian cohort. Indian J Endocrinol Metab 2013;17:381-2. 